Supporting Material for:

\title{
The Gas Phase Chemistry in Gallium Nitride CVD: Theoretical determination of the Arrhenius Parameters for the first Ga-C Bond Homolysis of Trimethylgallium
}

\author{
Rochus Schmid* and Daniel Basting \\ Lehrstuhl für Anorganische Chemie II, Organometallics and Materials Chemistry, Ruhr-Universität \\ Bochum, D-44780 Bochum, Germany \\ *rochus.schmid@ruhr-uni-bochum.de
}

Content:

1. Geometries of $\mathrm{GaMe}_{3}$ along the reaction coordinate

2. Calculated harmonic frequencies

3. Moments of Inertia

4. Energies used for the calculation of rate constants

5. Details on the calculation of the moments of inertia for the spectator methyl groups in fragment 1

6. Details on the calculation of the lambda parameter for the mixing of $\mathbf{m s}$ and $\mathbf{x} \mathbf{1}$

\section{Geometries of $\mathrm{GaMe}_{3}$ along the reaction coordinate}

$\mathrm{XYZ}$ coordinates of all atoms (in Angstroms) for all values of the reaction coordinate Ga-C used in the VTST calculation $(1.9 \AA-10.0 \AA)$.

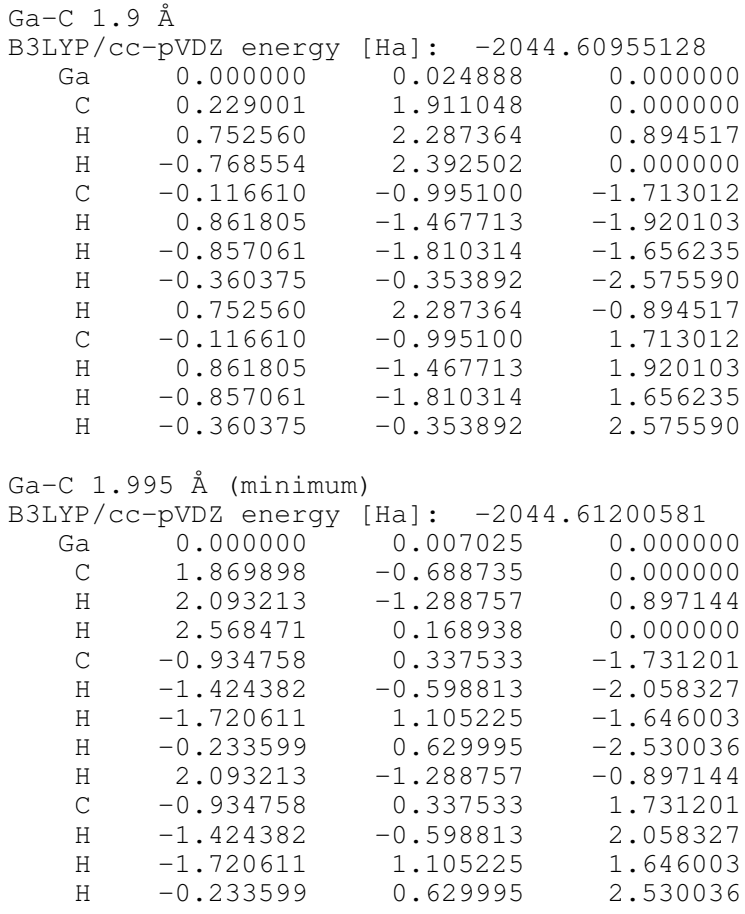


$\mathrm{Ga}-\mathrm{C} 2.2 \AA$

B3LYP/CC-pVDZ energy [Ha]: $\quad-2044.60457672$

$\begin{array}{rrrr}\mathrm{Ga} & 0.000000 & 0.052315 & 0.000000 \\ \mathrm{C} & 0.071595 & -2.146532 & 0.000000 \\ \mathrm{H} & -0.416917 & -2.544672 & 0.902545 \\ \mathrm{H} & 1.130039 & -2.457682 & 0.000000 \\ \mathrm{C} & -0.034890 & 0.989168 & -1.757833 \\ \mathrm{H} & -0.990222 & 0.774209 & -2.269106 \\ \mathrm{H} & 0.067878 & 2.081465 & -1.661505 \\ \mathrm{H} & 0.768796 & 0.611548 & -2.412590 \\ \mathrm{H} & -0.416917 & -2.544672 & -0.902545 \\ \mathrm{C} & -0.034890 & 0.989168 & 1.757833 \\ \mathrm{H} & -0.990222 & 0.774209 & 2.269106 \\ \mathrm{H} & 0.067878 & 2.081465 & 1.661505 \\ \mathrm{H} & 0.768796 & 0.611548 & 2.412590\end{array}$

$\mathrm{Ga}-\mathrm{C} 2.25 \AA$

B3LYP/CC-pVDZ energy [Ha]: $\quad-2044.60122779$

$\begin{array}{rrrr}\mathrm{Ga} & 0.000000 & 0.062513 & 0.000000 \\ \mathrm{C} & 0.030783 & -2.187289 & 0.000000 \\ \mathrm{H} & -0.468396 & -2.567695 & 0.903898 \\ \mathrm{H} & 1.083698 & -2.514457 & 0.000000 \\ \mathrm{C} & -0.015002 & 0.991683 & -1.761764 \\ \mathrm{H} & -0.957189 & 0.762890 & -2.290500 \\ \mathrm{H} & 0.075056 & 2.085298 & -1.667502 \\ \mathrm{H} & 0.806342 & 0.619554 & -2.397551 \\ \mathrm{H} & -0.468396 & -2.567695 & -0.903898 \\ \mathrm{C} & -0.015002 & 0.991683 & 1.761764 \\ \mathrm{H} & -0.957189 & 0.762890 & 2.290500 \\ \mathrm{H} & 0.075056 & 2.085298 & 1.667502 \\ \mathrm{H} & 0.806342 & 0.619554 & 2.397551\end{array}$

$\mathrm{Ga}-\mathrm{C} 2.3 \AA$

B3LYP/cC-pVDZ energy [Ha]: $\quad-2044.59752948$

$\begin{array}{rrrr}\mathrm{Ga} & 0.000000 & 0.072388 & 0.000000 \\ \mathrm{C} & 0.010051 & -2.227603 & 0.000000 \\ \mathrm{H} & -0.495525 & -2.594998 & 0.905255 \\ \mathrm{H} & 1.060590 & -2.560217 & 0.000000 \\ \mathrm{C} & -0.004978 & 0.994367 & -1.765275 \\ \mathrm{H} & -0.936781 & 0.751498 & -2.305500 \\ \mathrm{H} & 0.071719 & 2.089225 & -1.673305 \\ \mathrm{H} & 0.830010 & 0.628988 & -2.387039 \\ \mathrm{H} & -0.495525 & -2.594998 & -0.905255 \\ \mathrm{C} & -0.004978 & 0.994367 & 1.765275 \\ \mathrm{H} & -0.936781 & 0.751498 & 2.305500 \\ \mathrm{H} & 0.071719 & 2.089225 & 1.673305 \\ \mathrm{H} & 0.830010 & 0.628988 & 2.387039\end{array}$

$\mathrm{Ga}-\mathrm{C} 2.4 \AA$

B3LYP/CC-pVDZ energy [Ha]: $\quad-2044.58941898$

$\begin{array}{rrrr}\text { Ga } & 0.000000 & 0.091426 & 0.000000 \\ \mathrm{C} & -0.003681 & -2.308584 & 0.000000 \\ \mathrm{H} & -0.516361 & -2.656548 & 0.907967 \\ \mathrm{H} & 1.046716 & -2.637583 & 0.000000 \\ \mathrm{C} & 0.001588 & 1.000814 & -1.771435 \\ \mathrm{H} & -0.916270 & 0.734733 & -2.323683 \\ \mathrm{H} & 0.056323 & 2.097436 & -1.683867 \\ \mathrm{H} & 0.854466 & 0.646928 & -2.375133 \\ \mathrm{H} & -0.516361 & -2.656548 & -0.907967 \\ \mathrm{C} & 0.001588 & 1.000814 & 1.771435 \\ \mathrm{H} & -0.916270 & 0.734733 & 2.323683 \\ \mathrm{H} & 0.056323 & 2.097436 & 1.683867 \\ \mathrm{H} & 0.854466 & 0.646928 & 2.375133\end{array}$

$\mathrm{Ga}-\mathrm{C} 2.5 \AA$

B3LYP/CC-pVDZ energy [Ha]: $\quad-2044.58077932$

$\begin{array}{rrrr}\mathrm{Ga} & 0.000000 & 0.109723 & 0.000000 \\ \mathrm{C} & -0.005382 & -2.390284 & 0.000000 \\ \mathrm{H} & -0.522446 & -2.721758 & 0.910663 \\ \mathrm{H} & 1.047011 & -2.708892 & 0.000000 \\ \mathrm{C} & 0.002380 & 1.008621 & -1.776731 \\ \mathrm{H} & -0.907005 & 0.725932 & -2.334126 \\ \mathrm{H} & 0.041884 & 2.106279 & -1.692777 \\ \mathrm{H} & 0.865925 & 0.662396 & -2.369350 \\ \mathrm{H} & -0.522446 & -2.721758 & -0.910663 \\ \mathrm{C} & 0.002380 & 1.008621 & 1.776731 \\ \mathrm{H} & -0.907005 & 0.725932 & 2.334126 \\ \mathrm{H} & 0.041884 & 2.106279 & 1.692777 \\ \mathrm{H} & 0.865925 & 0.662396 & 2.369350\end{array}$

$\mathrm{Ga}-\mathrm{C} 2.6 \AA$

B3LYP/cC-pVDZ energy [Ha]: -2044.57197203

$\begin{array}{rrrr}\mathrm{Ga} & 0.000000 & 0.127386 & 0.000000 \\ \mathrm{C} & -0.009689 & -2.472610 & 0.000000 \\ \mathrm{H} & -0.530829 & -2.787241 & 0.913326 \\ \mathrm{H} & 1.044642 & -2.780913 & 0.000000 \\ \mathrm{C} & 0.004389 & 1.017634 & -1.781298 \\ \mathrm{H} & -0.896783 & 0.720480 & -2.343933 \\ \mathrm{H} & 0.030259 & 2.116005 & -1.699980 \\ \mathrm{H} & 0.877765 & 0.678765 & -2.363452 \\ \mathrm{H} & -0.530829 & -2.787241 & -0.913326 \\ \mathrm{C} & 0.004389 & 1.017634 & 1.781298 \\ \mathrm{H} & -0.896783 & 0.720480 & 2.343933 \\ \mathrm{H} & 0.030259 & 2.116005 & 1.699980 \\ \mathrm{H} & 0.877765 & 0.678765 & 2.363452\end{array}$

$\mathrm{Ga}-\mathrm{C} 2.7 \AA$

B3LYP/cC-pVDZ energy [Ha]: $\quad-2044.56324037$

$\begin{array}{rrrr}\mathrm{Ga} & 0.000000 & 0.144450 & 0.000000 \\ \mathrm{C} & -0.002423 & -2.555564 & 0.000000 \\ \mathrm{H} & -0.525778 & -2.855779 & 0.915937 \\ \mathrm{H} & 1.055336 & -2.848103 & 0.000000 \\ \mathrm{C} & 0.000999 & 1.027791 & -1.785192 \\ \mathrm{H} & -0.899372 & 0.724687 & -2.345589 \\ \mathrm{H} & 0.022735 & 2.126498 & -1.705680 \\ \mathrm{H} & 0.876020 & 0.689623 & -2.364939 \\ \mathrm{H} & -0.525778 & -2.855779 & -0.915937 \\ \mathrm{C} & 0.000999 & 1.027791 & 1.785192 \\ \mathrm{H} & -0.899372 & 0.724687 & 2.345589 \\ \mathrm{H} & 0.022735 & 2.126498 & 1.705680 \\ \mathrm{H} & 0.876020 & 0.689623 & 2.364939\end{array}$

$\mathrm{Ga}-\mathrm{C} 2.75 \AA$

B3LYP/cC-pVDZ energy [Ha]: $\quad-2044.55895526$

$\begin{array}{rrrr}\mathrm{Ga} & 0.000000 & 0.152785 & 0.000000 \\ \mathrm{C} & -0.002018 & -2.597229 & 0.000000 \\ \mathrm{H} & -0.526728 & -2.889618 & 0.917217 \\ \mathrm{H} & 1.057094 & -2.882985 & 0.000000 \\ \mathrm{C} & 0.000816 & 1.033250 & -1.786917 \\ \mathrm{H} & -0.897974 & 0.726546 & -2.347706 \\ \mathrm{H} & 0.019444 & 2.132113 & -1.707973 \\ \mathrm{H} & 0.877866 & 0.696478 & -2.364278 \\ \mathrm{H} & -0.526728 & -2.889618 & -0.917217 \\ \mathrm{C} & 0.000816 & 1.033250 & 1.786917 \\ \mathrm{H} & -0.897974 & 0.726546 & 2.347706 \\ \mathrm{H} & 0.019444 & 2.132113 & 1.707973 \\ \mathrm{H} & 0.877866 & 0.696478 & 2.364278\end{array}$

$\mathrm{Ga}-\mathrm{C} 2.8 \AA$

B3LYP/CC-pVDZ energy [Ha]: -2044.55474395

\begin{tabular}{rrrr}
$\mathrm{Ga}$ & 0.000000 & \multicolumn{1}{c}{0.160986} & 0.000000 \\
$\mathrm{C}$ & -0.004573 & -2.639025 & 0.000000 \\
$\mathrm{H}$ & -0.530862 & -2.923038 & 0.918477 \\
$\mathrm{H}$ & 1.055596 & -2.919011 & 0.000000 \\
$\mathrm{C}$ & 0.001999 & 1.038973 & -1.788482 \\
$\mathrm{H}$ & -0.893542 & 0.727229 & -2.351476 \\
$\mathrm{H}$ & 0.015577 & 2.137989 & -1.709878 \\
$\mathrm{H}$ & 0.882754 & 0.705282 & -2.361902 \\
$\mathrm{H}$ & -0.530862 & -2.923038 & -0.918477 \\
$\mathrm{C}$ & 0.001999 & 1.038973 & 1.788482 \\
$\mathrm{H}$ & -0.893542 & 0.727229 & 2.351476 \\
$\mathrm{H}$ & 0.015577 & 2.137989 & 1.709878 \\
$\mathrm{H}$ & 0.882754 & 0.705282 & 2.361902
\end{tabular}

$\mathrm{Ga}-\mathrm{C} 2.9 \AA$

B3LYP/CC-pVDZ energy [Ha]: $\quad-2044.54658494$

$\begin{array}{rrrr}\mathrm{Ga} & 0.000000 & 0.177081 & 0.000000 \\ \mathrm{C} & -0.001218 & -2.722935 & 0.000000 \\ \mathrm{H} & -0.529544 & -2.991910 & 0.920927 \\ \mathrm{H} & 1.061916 & -2.987830 & 0.000000 \\ \mathrm{C} & 0.000460 & 1.051011 & -1.791291 \\ \mathrm{H} & -0.894627 & 0.735950 & -2.352884 \\ \mathrm{H} & 0.011771 & 2.150192 & -1.712990 \\ \mathrm{H} & 0.882335 & 0.717664 & -2.362922 \\ \mathrm{H} & -0.529544 & -2.991910 & -0.920927 \\ \mathrm{C} & 0.000460 & 1.051011 & 1.791291 \\ \mathrm{H} & -0.894627 & 0.735950 & 2.352884 \\ \mathrm{H} & 0.011771 & 2.150192 & 1.712990 \\ \mathrm{H} & 0.882335 & 0.717664 & 2.362922 \\ & & & \end{array}$


$\mathrm{Ga}-\mathrm{C} 3.0 \AA$

B3LYP/CC-pVDZ energy [Ha]: $\quad-2044.53882493$

$\begin{array}{rrrr}\mathrm{Ga} & 0.000000 & 0.192738 & 0.000000 \\ \mathrm{C} & 0.000744 & -2.807279 & 0.000000 \\ \mathrm{H} & -0.529500 & -3.061094 & 0.923273 \\ \mathrm{H} & 1.066644 & -3.057285 & 0.000000 \\ \mathrm{C} & -0.000412 & 1.063915 & -1.793596 \\ \mathrm{H} & -0.895620 & 0.747244 & -2.353857 \\ \mathrm{H} & 0.010135 & 2.163189 & -1.714898 \\ \mathrm{H} & 0.881902 & 0.730207 & -2.364104 \\ \mathrm{H} & -0.529500 & -3.061094 & -0.923273 \\ \mathrm{C} & -0.000412 & 1.063915 & 1.793596 \\ \mathrm{H} & -0.895620 & 0.747244 & 2.353857 \\ \mathrm{H} & 0.010135 & 2.163189 & 1.714898 \\ \mathrm{H} & 0.881902 & 0.730207 & 2.364104\end{array}$

$\mathrm{Ga}-\mathrm{C} 3.05 \AA$

B3LYP/CC-pVDZ energy [Ha]: $\quad-2044.53510577$

$\begin{array}{rrrr}\mathrm{Ga} & 0.000000 & 0.200440 & 0.000000 \\ \mathrm{C} & 0.001376 & -2.849576 & 0.000000 \\ \mathrm{H} & -0.529782 & -3.095784 & 0.924401 \\ \mathrm{H} & 1.068585 & -3.092222 & 0.000000 \\ \mathrm{C} & -0.000690 & 1.070623 & -1.794600 \\ \mathrm{H} & -0.895822 & 0.753173 & -2.354438 \\ \mathrm{H} & 0.009348 & 2.169933 & -1.715574 \\ \mathrm{H} & 0.881975 & 0.736957 & -2.364484 \\ \mathrm{H} & -0.529782 & -3.095784 & -0.924401 \\ \mathrm{C} & -0.000690 & 1.070623 & 1.794600 \\ \mathrm{H} & -0.895822 & 0.753173 & 2.354438 \\ \mathrm{H} & 0.009348 & 2.169933 & 1.715574 \\ \mathrm{H} & 0.881975 & 0.736957 & 2.364484\end{array}$

$\mathrm{Ga}-\mathrm{C} 3.1 \AA$

B3LYP/cC-pVDZ energy [Ha]: $\quad-2044.53149702$

$\begin{array}{rrrr}\mathrm{Ga} & 0.000000 & 0.208053 & 0.000000 \\ \mathrm{C} & 0.001881 & -2.891963 & 0.000000 \\ \mathrm{H} & -0.530147 & -3.130600 & 0.925496 \\ \mathrm{H} & 1.070362 & -3.127263 & 0.000000 \\ \mathrm{C} & -0.000906 & 1.077509 & -1.795502 \\ \mathrm{H} & -0.896114 & 0.759645 & -2.354885 \\ \mathrm{H} & 0.008935 & 2.176841 & -1.715979 \\ \mathrm{H} & 0.881939 & 0.743754 & -2.364958 \\ \mathrm{H} & -0.530147 & -3.130600 & -0.925496 \\ \mathrm{C} & -0.000906 & 1.077509 & 1.795502 \\ \mathrm{H} & -0.896114 & 0.759645 & 2.354885 \\ \mathrm{H} & 0.008935 & 2.176841 & 1.715979 \\ \mathrm{H} & 0.881939 & 0.743754 & 2.364958\end{array}$

$\mathrm{Ga}-\mathrm{C} 3.15 \AA$

B3LYP/CC-pVDZ energy [Ha]: $\quad-2044.52799968$

$\begin{array}{rrrr}\text { Ga } & 0.000000 & 0.215607 & 0.000000 \\ \mathrm{C} & 0.002273 & -2.934409 & 0.000000 \\ \mathrm{H} & -0.530583 & -3.165515 & 0.926557 \\ \mathrm{H} & 1.071984 & -3.162401 & 0.000000 \\ \mathrm{C} & -0.001071 & 1.084523 & -1.796332 \\ \mathrm{H} & -0.896373 & 0.766357 & -2.355303 \\ \mathrm{H} & 0.008654 & 2.183870 & -1.716268 \\ \mathrm{H} & 0.881914 & 0.750665 & -2.365422 \\ \mathrm{H} & -0.530583 & -3.165515 & -0.926557 \\ \mathrm{C} & -0.001071 & 1.084523 & 1.796332 \\ \mathrm{H} & -0.896373 & 0.766357 & 2.355303 \\ \mathrm{H} & 0.008654 & 2.183870 & 1.716268 \\ \mathrm{H} & 0.881914 & 0.750665 & 2.365422\end{array}$

$\mathrm{Ga}-\mathrm{C} 3.2 \AA$

B3LYP/CC-pVDZ energy [Ha]: $\quad-2044.52461383$

$\begin{array}{rrrr}\mathrm{Ga} & 0.000000 & 0.223089 & 0.000000 \\ \mathrm{C} & 0.002570 & -2.976927 & 0.000000 \\ \mathrm{H} & -0.531083 & -3.200536 & 0.927581 \\ \mathrm{H} & 1.073463 & -3.197674 & 0.000000 \\ \mathrm{C} & -0.001190 & 1.091689 & -1.797070 \\ \mathrm{H} & -0.896609 & 0.773370 & -2.355688 \\ \mathrm{H} & 0.008512 & 2.191038 & -1.716349 \\ \mathrm{H} & 0.881880 & 0.757725 & -2.365885 \\ \mathrm{H} & -0.531083 & -3.200536 & -0.927581 \\ \mathrm{C} & -0.001190 & 1.091689 & 1.797070 \\ \mathrm{H} & -0.896609 & 0.773370 & 2.355688 \\ \mathrm{H} & 0.008512 & 2.191038 & 1.716349 \\ \mathrm{H} & 0.881880 & 0.757725 & 2.365885\end{array}$

$\mathrm{Ga}-\mathrm{C} 3.25 \AA$

B3LYP/CC-pVDZ energy [Ha]: -2044.52148753

$\begin{array}{rrrr}\mathrm{Ga} & 0.000000 & 0.224970 & 0.000000 \\ \mathrm{C} & 0.003187 & -3.025046 & 0.000000 \\ \mathrm{H} & -0.531868 & -3.234005 & 0.929714 \\ \mathrm{H} & 1.076571 & -3.231118 & 0.000000 \\ \mathrm{C} & -0.001459 & 1.108893 & -1.793422 \\ \mathrm{H} & -0.897123 & 0.796208 & -2.354939 \\ \mathrm{H} & 0.008355 & 2.207580 & -1.702366 \\ \mathrm{H} & 0.881542 & 0.780510 & -2.365691 \\ \mathrm{H} & -0.531868 & -3.234005 & -0.929714 \\ \mathrm{C} & -0.001459 & 1.108893 & 1.793422 \\ \mathrm{H} & -0.897123 & 0.796208 & 2.354939 \\ \mathrm{H} & 0.008355 & 2.207580 & 1.702366 \\ \mathrm{H} & 0.881542 & 0.780510 & 2.365691\end{array}$

$\mathrm{Ga}-\mathrm{C} 3.3 \AA$

B3LYP/cC-pVDZ energy [Ha]: $\quad-2044.51872914$

$\begin{array}{rrrr}\mathrm{Ga} & 0.000000 & 0.227587 & 0.000000 \\ \mathrm{C} & 0.003260 & -3.072429 & 0.000000 \\ \mathrm{H} & -0.533082 & -3.267291 & 0.931622 \\ \mathrm{H} & 1.078835 & -3.264659 & 0.000000 \\ \mathrm{C} & -0.001478 & 1.124737 & -1.790249 \\ \mathrm{H} & -0.897101 & 0.816705 & -2.354498 \\ \mathrm{H} & 0.008153 & 2.222779 & -1.690290 \\ \mathrm{H} & 0.881701 & 0.801399 & -2.365209 \\ \mathrm{H} & -0.533082 & -3.267291 & -0.931622 \\ \mathrm{C} & -0.001478 & 1.124737 & 1.790249 \\ \mathrm{H} & -0.897101 & 0.816705 & 2.354498 \\ \mathrm{H} & 0.008153 & 2.222779 & 1.690290 \\ \mathrm{H} & 0.881701 & 0.801399 & 2.365209\end{array}$

$\mathrm{Ga}-\mathrm{C} 3.4 \AA$

B3LYP/cC-pVDZ energy [Ha]: -2044.51418189

$\begin{array}{rrrr}\mathrm{Ga} & 0.000000 & 0.213469 & 0.000000 \\ \mathrm{C} & 0.000816 & -3.186549 & 0.000000 \\ \mathrm{H} & -0.538157 & -3.350435 & 0.935304 \\ \mathrm{H} & 1.080488 & -3.349781 & 0.000000 \\ \mathrm{C} & -0.000390 & 1.185334 & -1.758935 \\ \mathrm{H} & -0.896682 & 1.828423 & -1.812868 \\ \mathrm{H} & 0.890137 & 1.836638 & -1.809268 \\ \mathrm{H} & 0.004347 & 0.499143 & -2.617296 \\ \mathrm{H} & -0.538157 & -3.350435 & -0.935304 \\ \mathrm{C} & -0.000390 & 1.185334 & 1.758935 \\ \mathrm{H} & -0.896682 & 1.828423 & 1.812868 \\ \mathrm{H} & 0.890137 & 1.836638 & 1.809268 \\ \mathrm{H} & 0.004347 & 0.499143 & 2.617296\end{array}$

$\mathrm{Ga}-\mathrm{C} 3.5 \AA$

B3LYP/CC-pVDZ energy [Ha]: $\quad-2044.51068058$

$\begin{array}{rrrr}\mathrm{Ga} & 0.000000 & 0.222567 & 0.000000 \\ \mathrm{C} & 0.001769 & -3.277452 & 0.000000 \\ \mathrm{H} & -0.538826 & -3.417131 & 0.937791 \\ \mathrm{H} & 1.084355 & -3.416598 & 0.000000 \\ \mathrm{C} & -0.000822 & 1.210019 & -1.754404 \\ \mathrm{H} & -0.897524 & 1.853221 & -1.801723 \\ \mathrm{H} & 0.889741 & 1.861913 & -1.798168 \\ \mathrm{H} & 0.004056 & 0.532754 & -2.619921 \\ \mathrm{H} & -0.538826 & -3.417131 & -0.937791 \\ \mathrm{C} & -0.000822 & 1.210019 & 1.754404 \\ \mathrm{H} & -0.897524 & 1.853221 & 1.801723 \\ \mathrm{H} & 0.889741 & 1.861913 & 1.798168 \\ \mathrm{H} & 0.004056 & 0.532754 & 2.619921\end{array}$

$\mathrm{Ga}-\mathrm{C} 3.6 \AA$

B3LYP/cC-pVDZ energy [Ha]: $\quad-2044.50791839$

$\begin{array}{rrrr}\mathrm{Ga} & 0.000000 & 0.232855 & 0.000000 \\ \mathrm{C} & 0.002829 & -3.367164 & 0.000000 \\ \mathrm{H} & -0.539037 & -3.484419 & 0.939750 \\ \mathrm{H} & 1.087717 & -3.483893 & 0.000000 \\ \mathrm{C} & -0.001307 & 1.232579 & -1.750779 \\ \mathrm{H} & -0.898413 & 1.875708 & -1.792896 \\ \mathrm{H} & 0.889183 & 1.885024 & -1.789382 \\ \mathrm{H} & 0.003766 & 0.562386 & -2.621864 \\ \mathrm{H} & -0.539037 & -3.484419 & -0.939750 \\ \mathrm{C} & -0.001307 & 1.232579 & 1.750779 \\ \mathrm{H} & -0.898413 & 1.875708 & 1.792896 \\ \mathrm{H} & 0.889183 & 1.885024 & 1.789382 \\ \mathrm{H} & 0.003766 & 0.562386 & 2.621864\end{array}$


$\mathrm{Ga}-\mathrm{C} 3.7 \AA$

B3LYP/CC-pVDZ energy [Ha]: $\quad-2044.50572730$

$\begin{array}{rrrr}\text { Ga } & 0.000000 & 0.244081 & 0.000000 \\ \mathrm{C} & 0.006578 & -3.455933 & 0.000000 \\ \mathrm{H} & -0.536200 & -3.552997 & 0.941250 \\ \mathrm{H} & 1.093299 & -3.551301 & 0.000000 \\ \mathrm{C} & -0.003066 & 1.253534 & -1.747844 \\ \mathrm{H} & -0.901166 & 1.895639 & -1.785675 \\ \mathrm{H} & 0.886668 & 1.907330 & -1.782509 \\ \mathrm{H} & 0.002712 & 0.589003 & -2.623319 \\ \mathrm{H} & -0.536200 & -3.552997 & -0.941250 \\ \mathrm{C} & -0.003066 & 1.253534 & 1.747844 \\ \mathrm{H} & -0.901166 & 1.895639 & 1.785675 \\ \mathrm{H} & 0.886668 & 1.907330 & 1.782509 \\ \mathrm{H} & 0.002712 & 0.589003 & 2.623319\end{array}$

$\mathrm{Ga}-\mathrm{C} 3.75 \AA$

B3LYP/CC-pVDZ energy [Ha]: $\quad-2044.50480463$

$\begin{array}{rrrr}\mathrm{Ga} & 0.000000 & 0.249989 & 0.000000 \\ \mathrm{C} & 0.002898 & -3.500030 & 0.000000 \\ \mathrm{H} & -0.540373 & -3.586959 & 0.941851 \\ \mathrm{H} & 1.090228 & -3.587009 & 0.000000 \\ \mathrm{C} & -0.001348 & 1.263537 & -1.746589 \\ \mathrm{H} & -0.897864 & 1.908006 & -1.782341 \\ \mathrm{H} & 0.890093 & 1.915120 & -1.779880 \\ \mathrm{H} & 0.002420 & 0.601380 & -2.623899 \\ \mathrm{H} & -0.540373 & -3.586959 & -0.941851 \\ \mathrm{C} & -0.001348 & 1.263537 & 1.746589 \\ \mathrm{H} & -0.897864 & 1.908006 & 1.782341 \\ \mathrm{H} & 0.890093 & 1.915120 & 1.779880 \\ \mathrm{H} & 0.002420 & 0.601380 & 2.623899\end{array}$

$\mathrm{Ga}-\mathrm{C} 3.8 \AA$

B3LYP/cC-pVDZ energy [Ha]: $\quad-2044.50397930$

$\begin{array}{rrrr}\text { Ga } & 0.000000 & 0.256068 & 0.000000 \\ \mathrm{C} & 0.005478 & -3.543950 & 0.000000 \\ \mathrm{H} & -0.538087 & -3.622148 & 0.942362 \\ \mathrm{H} & 1.093455 & -3.621311 & 0.000000 \\ \mathrm{C} & -0.002535 & 1.273272 & -1.745458 \\ \mathrm{H} & -0.900436 & 1.915914 & -1.780093 \\ \mathrm{H} & 0.887585 & 1.926787 & -1.776780 \\ \mathrm{H} & 0.002986 & 0.613270 & -2.624406 \\ \mathrm{H} & -0.538087 & -3.622148 & -0.942362 \\ \mathrm{C} & -0.002535 & 1.273272 & 1.745458 \\ \mathrm{H} & -0.900436 & 1.915914 & 1.780093 \\ \mathrm{H} & 0.887585 & 1.926787 & 1.776780 \\ \mathrm{H} & 0.002986 & 0.613270 & 2.624406\end{array}$

$\mathrm{Ga}-\mathrm{C} 3.85 \AA$

B3LYP/CC-pVDZ energy [Ha]: $\quad-2044.50323995$

$\begin{array}{rrrr}\mathrm{Ga} & 0.000000 & 0.262300 & 0.000000 \\ \mathrm{C} & 0.007470 & -3.587713 & 0.000000 \\ \mathrm{H} & -0.536362 & -3.657609 & 0.942794 \\ \mathrm{H} & 1.095976 & -3.656252 & 0.000000 \\ \mathrm{C} & -0.003498 & 1.282781 & -1.744436 \\ \mathrm{H} & -0.901053 & 1.926040 & -1.776962 \\ \mathrm{H} & 0.887049 & 1.935784 & -1.775098 \\ \mathrm{H} & 0.000956 & 0.624701 & -2.624853 \\ \mathrm{H} & -0.536362 & -3.657609 & -0.942794 \\ \mathrm{C} & -0.003498 & 1.282781 & 1.744436 \\ \mathrm{H} & -0.901053 & 1.926040 & 1.776962 \\ \mathrm{H} & 0.887049 & 1.935784 & 1.775098 \\ \mathrm{H} & 0.000956 & 0.624701 & 2.624853\end{array}$

$\mathrm{Ga}-\mathrm{C} 3.9 \AA$

B3LYP/CC-pVDZ energy [Ha]: $\quad-2044.50257664$

$\begin{array}{rrrr}\mathrm{Ga} & 0.000000 & 0.268676 & 0.000000 \\ \mathrm{C} & 0.007779 & -3.631337 & 0.000000 \\ \mathrm{H} & -0.536305 & -3.693271 & 0.943153 \\ \mathrm{H} & 1.096700 & -3.692088 & 0.000000 \\ \mathrm{C} & -0.003608 & 1.292093 & -1.743513 \\ \mathrm{H} & -0.902147 & 1.934042 & -1.775469 \\ \mathrm{H} & 0.885997 & 1.946488 & -1.772277 \\ \mathrm{H} & 0.002417 & 0.635754 & -2.625241 \\ \mathrm{H} & -0.536305 & -3.693271 & -0.943153 \\ \mathrm{C} & -0.003608 & 1.292093 & 1.743513 \\ \mathrm{H} & -0.902147 & 1.934042 & 1.775469 \\ \mathrm{H} & 0.885997 & 1.946488 & 1.772277 \\ \mathrm{H} & 0.002417 & 0.635754 & 2.625241\end{array}$

$\mathrm{Ga}-\mathrm{C} 3.95 \AA$

B3LYP/CC-pVDZ energy [Ha]: -2044.50198071

$\begin{array}{rrrr}\mathrm{Ga} & 0.000000 & 0.275185 & 0.000000 \\ \mathrm{C} & 0.012870 & -3.674816 & 0.000000 \\ \mathrm{H} & -0.531366 & -3.729978 & 0.943449 \\ \mathrm{H} & 1.102189 & -3.727191 & 0.000000 \\ \mathrm{C} & -0.006000 & 1.301225 & -1.742680 \\ \mathrm{H} & -0.905768 & 1.941539 & -1.773295 \\ \mathrm{H} & 0.882396 & 1.957325 & -1.770557 \\ \mathrm{H} & 0.001030 & 0.646454 & -2.625584 \\ \mathrm{H} & -0.531366 & -3.729978 & -0.943449 \\ \mathrm{C} & -0.006000 & 1.301225 & 1.742680 \\ \mathrm{H} & -0.905768 & 1.941539 & 1.773295 \\ \mathrm{H} & 0.882396 & 1.957325 & 1.770557 \\ \mathrm{H} & 0.001030 & 0.646454 & 2.625584\end{array}$

$\mathrm{Ga}-\mathrm{C} 4.0 \AA$

B3LYP/cC-pVDZ energy [Ha]: $\quad-2044.50144456$

$\begin{array}{rrrr}\text { Ga } & 0.000000 & 0.281801 & 0.000000 \\ \mathrm{C} & 0.011197 & -3.718205 & 0.000000 \\ \mathrm{H} & -0.533244 & -3.766308 & 0.943691 \\ \mathrm{H} & 1.100766 & -3.764517 & 0.000000 \\ \mathrm{C} & -0.005208 & 1.310239 & -1.741910 \\ \mathrm{H} & -0.904582 & 1.951175 & -1.771606 \\ \mathrm{H} & 0.883643 & 1.965790 & -1.768708 \\ \mathrm{H} & 0.001458 & 0.656877 & -2.625880 \\ \mathrm{H} & -0.533244 & -3.766308 & -0.943691 \\ \mathrm{C} & -0.005208 & 1.310239 & 1.741910 \\ \mathrm{H} & -0.904582 & 1.951175 & 1.771606 \\ \mathrm{H} & 0.883643 & 1.965790 & 1.768708 \\ \mathrm{H} & 0.001458 & 0.656877 & 2.625880\end{array}$

$\mathrm{Ga}-\mathrm{C} 4.1 \AA$

B3LYP/cC-pVDZ energy [Ha]: $\quad-2044.50052578$

$\begin{array}{rrrr}\mathrm{Ga} & 0.000000 & 0.295352 & 0.000000 \\ \mathrm{C} & 0.014238 & -3.804646 & 0.000000 \\ \mathrm{H} & -0.530452 & -3.841214 & 0.944039 \\ \mathrm{H} & 1.104221 & -3.839038 & 0.000000 \\ \mathrm{C} & -0.006633 & 1.327893 & -1.740576 \\ \mathrm{H} & -0.906673 & 1.968015 & -1.768378 \\ \mathrm{H} & 0.881606 & 1.984375 & -1.765834 \\ \mathrm{H} & 0.000490 & 0.676974 & -2.626375 \\ \mathrm{H} & -0.530452 & -3.841214 & -0.944039 \\ \mathrm{C} & -0.006633 & 1.327893 & 1.740576 \\ \mathrm{H} & -0.906673 & 1.968015 & 1.768378 \\ \mathrm{H} & 0.881606 & 1.984375 & 1.765834 \\ \mathrm{H} & 0.000490 & 0.676974 & 2.626375\end{array}$

$\mathrm{Ga}-\mathrm{C} 4.3 \stackrel{\circ}{\AA}$

B3LYP/cC-pVDZ energy [Ha]: $\quad-2044.49916218$

$\begin{array}{rrrr}\mathrm{Ga} & 0.000000 & 0.323460 & 0.000000 \\ \mathrm{C} & 0.032770 & -3.976439 & 0.000000 \\ \mathrm{H} & -0.512134 & -3.998069 & 0.944389 \\ \mathrm{H} & 1.123213 & -3.990040 & 0.000000 \\ \mathrm{C} & -0.015285 & 1.362137 & -1.738504 \\ \mathrm{H} & -0.920929 & 1.994470 & -1.763931 \\ \mathrm{H} & 0.867216 & 2.026467 & -1.760973 \\ \mathrm{H} & -0.002358 & 0.715009 & -2.627062 \\ \mathrm{H} & -0.512134 & -3.998069 & -0.944389 \\ \mathrm{C} & -0.015285 & 1.362137 & 1.738504 \\ \mathrm{H} & -0.920929 & 1.994470 & 1.763931 \\ \mathrm{H} & 0.867216 & 2.026467 & 1.760973 \\ \mathrm{H} & -0.002358 & 0.715009 & 2.627062\end{array}$

$\mathrm{Ga}-\mathrm{C} 4.5 \AA$

B3LYP/cC-pVDZ energy [Ha]: $\quad-2044.49824122$

$\begin{array}{rrrr}\mathrm{Ga} & 0.000000 & 0.352738 & 0.000000 \\ \mathrm{C} & 0.116940 & -4.145768 & 0.000000 \\ \mathrm{H} & -0.427804 & -4.167564 & 0.944522 \\ \mathrm{H} & 1.207350 & -4.128043 & 0.000000 \\ \mathrm{C} & -0.054615 & 1.394447 & -1.736996 \\ \mathrm{H} & -0.984279 & 1.991002 & -1.761284 \\ \mathrm{H} & 0.801279 & 2.092829 & -1.757040 \\ \mathrm{H} & -0.016004 & 0.750940 & -2.627476 \\ \mathrm{H} & -0.427804 & -4.167564 & -0.944522 \\ \mathrm{C} & -0.054615 & 1.394447 & 1.736996 \\ \mathrm{H} & -0.984279 & 1.991002 & 1.761284 \\ \mathrm{H} & 0.801279 & 2.092829 & 1.757040 \\ \mathrm{H} & -0.016004 & 0.750940 & 2.627476\end{array}$


$\mathrm{Ga}-\mathrm{C} 4.75 \AA$

B3LYP/CC-pVDZ energy [Ha]: $\quad-2044.49748847$

$\mathrm{Ga}$
$\mathrm{C}$
$\mathrm{H}$
$\mathrm{H}$
$\mathrm{C}$
$\mathrm{H}$
$\mathrm{H}$
$\mathrm{H}$
$\mathrm{H}$
$\mathrm{C}$
$\mathrm{H}$
$\mathrm{H}$
$\mathrm{H}$

$\begin{array}{rrr}0.000000 & 0.391174 & 0.000000 \\ 0.280950 & -4.350536 & 0.000000 \\ -0.262981 & -4.387244 & 0.944586 \\ 1.369382 & -4.282010 & 0.000000 \\ -0.131324 & 1.429687 & -1.735674 \\ -1.101175 & 1.958541 & -1.757593 \\ 0.672596 & 2.187366 & -1.755148 \\ -0.048029 & 0.792634 & -2.627772 \\ -0.262981 & -4.387244 & -0.944586 \\ -0.131324 & 1.429687 & 1.735674 \\ -1.101175 & 1.958541 & 1.757593 \\ 0.672596 & 2.187366 & 1.755148 \\ -0.048029 & 0.792634 & 2.627772\end{array}$

$\mathrm{Ga}-\mathrm{C} 10.0 \AA$

B3LYP/CC-pVDZ energy [Ha]: $\quad-2044.49631864$

$\begin{array}{rrrr}\mathrm{Ga} & 0.000000 & 1.368291 & 0.000000 \\ \mathrm{C} & 2.026728 & -8.424231 & 0.000000 \\ \mathrm{H} & 1.525713 & -8.639935 & 0.944576 \\ \mathrm{H} & 3.028542 & -7.992825 & 0.000000 \\ \mathrm{C} & -0.947552 & 1.823936 & -1.734022 \\ \mathrm{H} & -1.920451 & 1.300580 & -1.752405 \\ \mathrm{H} & -1.142801 & 2.911260 & -1.753918 \\ \mathrm{H} & -0.371606 & 1.545073 & -2.628022 \\ \mathrm{H} & 1.525713 & -8.639935 & -0.944576 \\ \mathrm{C} & -0.947552 & 1.823936 & 1.734022 \\ \mathrm{H} & -1.920451 & 1.300580 & 1.752405 \\ \mathrm{H} & -1.142801 & 2.911260 & 1.753918 \\ \mathrm{H} & -0.371606 & 1.545073 & 2.628022\end{array}$

$\mathrm{Ga}-\mathrm{C} 5.0 \AA$

B3LYP/CC-pVDZ energy [Ha]: -2044.49702628

$\begin{array}{rrrr}\mathrm{Ga} & 0.000000 & 0.484779 & 0.000000 \\ \mathrm{C} & 1.536495 & -4.273315 & 0.000000 \\ \mathrm{H} & 1.041717 & -4.502522 & 0.944650 \\ \mathrm{H} & 2.526250 & -3.815297 & 0.000000 \\ \mathrm{C} & -0.718554 & 1.248532 & -1.735146 \\ \mathrm{H} & -1.815687 & 1.118996 & -1.751069 \\ \mathrm{H} & -0.500901 & 2.331533 & -1.759579 \\ \mathrm{H} & -0.286417 & 0.774323 & -2.627942 \\ \mathrm{H} & 1.041717 & -4.502522 & -0.944650 \\ \mathrm{C} & -0.718554 & 1.248532 & 1.735146 \\ \mathrm{H} & -1.815687 & 1.118996 & 1.751069 \\ \mathrm{H} & -0.500901 & 2.331533 & 1.759579 \\ \mathrm{H} & -0.286417 & 0.774323 & 2.627942\end{array}$

\section{Calculated harmonic frequencies}

All frequencies are given in $1 / \mathrm{cm}$. The first eight frequencies have been assigned.

\section{Table 2.1}

\begin{tabular}{|c|c|c|c|c|c|c|c|c|c|c|c|c|c|c|c|c|}
\hline rc & rc & $\mathbf{z}$ & $\mathbf{x 1}$ & y1 & $\times 2$ & y2 & ms & ma & & & & & & & & \\
\hline 1.900 & 662 & 33 & 133 & 132 & 593 & 657 & -9 & -28 & 157 & 513 & 548 & 610 & 734 & 770 & 771 & 1211 \\
\hline 1.995 & 555 & 41 & 149 & 153 & 612 & 656 & 27 & -26 & 155 & 499 & 552 & 605 & 734 & 767 & 768 & 1210 \\
\hline 2.000 & 551 & 41 & 150 & 154 & 612 & 656 & 29 & -23 & 155 & 497 & 552 & 605 & 734 & 767 & 768 & 1209 \\
\hline 2.100 & 422 & -16 & 160 & 163 & 622 & 652 & 35 & 32 & 152 & 521 & 558 & 601 & 735 & 762 & 765 & 1174 \\
\hline 2.200 & 334 & 8 & 163 & 167 & 625 & 648 & 33 & 27 & 149 & 516 & 561 & 602 & 735 & 754 & 764 & 1140 \\
\hline 2.250 & 293 & 12 & 163 & 167 & 626 & 646 & 34 & 24 & 148 & 515 & 562 & 603 & 735 & 750 & 764 & 1123 \\
\hline 2.300 & 254 & 11 & 162 & 166 & 625 & 643 & 36 & 25 & 147 & 513 & 563 & 604 & 734 & 745 & 764 & 1106 \\
\hline 2.400 & 179 & 3 & 159 & 162 & 622 & 637 & 39 & 31 & 145 & 511 & 564 & 606 & 732 & 736 & 764 & 1072 \\
\hline 2.500 & 99 & -5 & 156 & 157 & 617 & 630 & 42 & 36 & 144 & 508 & 564 & 608 & 727 & 730 & 763 & 1038 \\
\hline 2.600 & -72 & -2 & 152 & 152 & 610 & 621 & 43 & 38 & 142 & 506 & 563 & 610 & 719 & 728 & 762 & 1005 \\
\hline 2.700 & -122 & 6 & 147 & 145 & 600 & 610 & 43 & 38 & 141 & 503 & 562 & 612 & 711 & 725 & 761 & 972 \\
\hline 2.750 & -136 & 7 & 145 & 142 & 594 & 604 & 43 & 38 & 140 & 502 & 561 & 613 & 708 & 724 & 760 & 955 \\
\hline 2.800 & -146 & 9 & 142 & 139 & 588 & 598 & 43 & 38 & 140 & 501 & 560 & 613 & 705 & 723 & 760 & 939 \\
\hline 2.900 & -158 & 11 & 137 & 132 & 574 & 586 & 41 & 37 & 138 & 498 & 557 & 614 & 699 & 721 & 758 & 907 \\
\hline 3.000 & -164 & 13 & 132 & 126 & 559 & 575 & 40 & 36 & 137 & 496 & 551 & 615 & 693 & 718 & 756 & 876 \\
\hline 3.050 & -165 & 14 & 130 & 122 & 551 & 546 & 39 & 35 & 137 & 494 & 571 & 615 & 691 & 717 & 755 & 861 \\
\hline 3.100 & -166 & 14 & 128 & 119 & 542 & 539 & 39 & 34 & 137 & 493 & 568 & 615 & 689 & 716 & 753 & 846 \\
\hline 3.150 & -166 & 15 & 125 & 116 & 534 & 532 & 38 & 34 & 136 & 492 & 565 & 616 & 687 & 715 & 752 & 832 \\
\hline 3.200 & -165 & 15 & 123 & 113 & 525 & 524 & 37 & 33 & 136 & 491 & 563 & 616 & 685 & 714 & 750 & 818 \\
\hline 3.250 & -298 & 16 & 113 & 105 & 500 & 500 & 29 & 24 & 137 & 486 & 557 & 613 & 677 & 705 & 729 & 782 \\
\hline 3.300 & -279 & 16 & 104 & 98 & 475 & 476 & 20 & 9 & 138 & 483 & 552 & 610 & 671 & 698 & 715 & 768 \\
\hline 3.400 & -241 & 15 & 94 & 86 & 423 & 425 & 20 & -24 & 138 & 479 & 534 & 606 & 614 & 666 & 673 & 770 \\
\hline 3.500 & -212 & 10 & 83 & 75 & 381 & 381 & 28 & -14 & 139 & 476 & 529 & 600 & 602 & 626 & 661 & 767 \\
\hline 3.600 & -186 & -3 & 73 & 66 & 342 & 342 & 31 & 10 & 140 & 473 & 525 & 583 & 590 & 598 & 656 & 766 \\
\hline 3.700 & -164 & -8 & 66 & 58 & 306 & 306 & 33 & 18 & 141 & 471 & 521 & 546 & 583 & 596 & 652 & 765 \\
\hline 3.800 & -144 & -8 & 61 & 51 & 274 & 273 & 33 & 22 & 141 & 470 & 516 & 519 & 578 & 593 & 649 & 764 \\
\hline 3.900 & -127 & -5 & 32 & 45 & 245 & 244 & 57 & 25 & 142 & 469 & 493 & 517 & 573 & 592 & 647 & 764 \\
\hline 4.000 & -112 & 5 & 29 & 39 & 219 & 219 & 55 & 27 & 142 & 468 & 477 & 515 & 570 & 590 & 645 & 763 \\
\hline 4.100 & -100 & 8 & 26 & 35 & 195 & 195 & 54 & 29 & 143 & 466 & 468 & 514 & 568 & 589 & 644 & 763 \\
\hline 4.300 & -80 & 9 & 20 & 27 & 154 & 156 & 54 & 31 & 144 & 459 & 467 & 512 & 564 & 587 & 642 & 763 \\
\hline 4.500 & -64 & 8 & 16 & 21 & 122 & 124 & 54 & 34 & 144 & 458 & 466 & 511 & 562 & 586 & 640 & 763 \\
\hline 4.750 & -50 & 6 & 11 & 15 & 89 & 91 & 56 & 36 & 145 & 460 & 466 & 510 & 560 & 585 & 639 & 763 \\
\hline 5.000 & -39 & 10 & 6 & 9 & 63 & 69 & 56 & 36 & 145 & 463 & 466 & 509 & 560 & 585 & 638 & 763 \\
\hline
\end{tabular}




\section{Table 2.2}

\begin{tabular}{|c|c|c|c|c|c|c|c|c|c|c|c|c|c|c|c|c|c|}
\hline & & & & & & & & & & & & & & & & & \\
\hline 1.900 & 1216 & 1249 & 1424 & 1424 & 1428 & 1430 & 1434 & 1439 & 3008 & 3013 & 3015 & 3075 & 3085 & 3086 & 3108 & 3114 & 3114 \\
\hline 1.995 & 1210 & 1216 & 1422 & 1425 & 1428 & 1429 & 1433 & 1437 & 3014 & 3014 & 3017 & 3088 & 3088 & 3089 & 3118 & 3118 & 3118 \\
\hline 2.000 & 1210 & 1216 & 1422 & 1426 & 1428 & 1429 & 1433 & 1437 & 3015 & 3015 & 3017 & 3088 & 3089 & 3089 & 3118 & 3118 & 3118 \\
\hline 2.100 & 1208 & 1213 & 1422 & 1425 & 1426 & 1430 & 1432 & 1435 & 3018 & 3018 & 3023 & 3091 & 3092 & 3104 & 3119 & 3120 & 3129 \\
\hline 2.200 & 1207 & 1212 & 1421 & 1424 & 1425 & 1429 & 1431 & 1433 & 3020 & 3021 & 3030 & 3095 & 3095 & 3119 & 3120 & 3121 & 3140 \\
\hline 2.250 & 1206 & 1211 & 1421 & 1423 & 1424 & 1429 & 1431 & 1433 & 3021 & 3022 & 3033 & 3097 & 3097 & 3120 & 3121 & 3127 & 3146 \\
\hline 2.300 & 1205 & 1210 & 1421 & 1422 & 1423 & 1428 & 1430 & 1432 & 3021 & 3022 & 3036 & 3098 & 3099 & 3121 & 3121 & 3135 & 3152 \\
\hline 2.400 & 1203 & 1208 & 1420 & 1420 & 1421 & 1426 & 1429 & 1430 & 3022 & 3023 & 3043 & 3102 & 3102 & 3121 & 3122 & 3150 & 3164 \\
\hline 2.500 & 1201 & 1207 & 1418 & 1418 & 1420 & 1424 & 1428 & 1429 & 3023 & 3024 & 3050 & 3105 & 3105 & 3122 & 3122 & 3165 & 3176 \\
\hline 2.600 & 1199 & 1205 & 1414 & 1415 & 1420 & 1423 & 1426 & 1429 & 3024 & 3025 & 3056 & 3107 & 3108 & 3122 & 3122 & 3180 & 3188 \\
\hline 2.700 & 1197 & 1203 & 1411 & 1412 & 1420 & 1421 & 1424 & 1428 & 3024 & 3025 & 3063 & 3110 & 3110 & 3122 & 3123 & 3194 & 3201 \\
\hline 2.750 & 1196 & 1202 & 1409 & 1410 & 1419 & 1420 & 1424 & 1428 & 3024 & 3025 & 3066 & 3111 & 3112 & 3122 & 3123 & 3200 & 3206 \\
\hline 2.800 & 1195 & 1201 & 1408 & 1408 & 1419 & 1420 & 1423 & 1428 & 3025 & 3025 & 3069 & 3112 & & 3122 & 3123 & 3207 & 3212 \\
\hline 2.900 & 1193 & 1200 & 1404 & 1405 & 1418 & 1419 & 1422 & 1427 & 3025 & 3026 & 3075 & 3114 & 15 & 3122 & 3123 & 3219 & 3224 \\
\hline 3.000 & 1191 & 1198 & 1401 & 1401 & 1417 & 1418 & 1420 & 1427 & 3025 & 3025 & 3081 & 3116 & 31 & 3122 & 3123 & 3231 & 3235 \\
\hline 3.050 & 1190 & 1197 & 1399 & 1399 & 1417 & 1418 & 1420 & 1427 & 3025 & 3025 & 3084 & 3116 & & 3122 & 3123 & 3237 & 3240 \\
\hline 3.100 & 1189 & 1196 & 1397 & 1398 & 1416 & 1418 & 1419 & 1427 & 3024 & 3025 & 3087 & 3117 & 3118 & 3122 & 3123 & 3242 & 3245 \\
\hline 3.150 & 1188 & 1196 & 1396 & 1396 & 1416 & 1418 & 1419 & 1427 & 3024 & 3025 & 3089 & 3118 & & 3122 & 3123 & 47 & 3250 \\
\hline 3.200 & 1187 & 1195 & 1394 & 1395 & 1415 & 1418 & 1418 & 1426 & 3024 & 3025 & 3092 & 3118 & & 3122 & 3123 & 3252 & 3254 \\
\hline 3 & 184 & 1193 & 1391 & 1391 & 1415 & 1417 & 1418 & 1426 & 3023 & 3024 & 3094 & 3117 & 3118 & 3121 & 3122 & 3259 & 3261 \\
\hline 3.300 & 1181 & 1191 & 1388 & 1388 & 1414 & 1417 & 1418 & 1426 & 3022 & 3023 & 3096 & 3117 & 31 & 3120 & 3120 & 3264 & 3266 \\
\hline 3.400 & 1172 & 1185 & 1382 & 1382 & 1409 & 1414 & 1421 & 1432 & 3015 & 3017 & 3096 & 3097 & & 3143 & 3143 & 3275 & 3277 \\
\hline 3.500 & 1168 & 1182 & 1378 & 1379 & 1409 & 1414 & 1421 & 1432 & 3014 & 3016 & 3095 & 3096 & 3102 & 3142 & 142 & 3282 & 3283 \\
\hline 3.600 & 1165 & 1180 & 1376 & 1376 & 1408 & 1414 & 1421 & 1432 & 3013 & 3015 & 3094 & 3096 & 31 & 41 & 3141 & 3287 & 3288 \\
\hline 3.700 & 1163 & 1179 & 1374 & 1374 & 1408 & 1413 & 1420 & 1432 & 3012 & 3014 & 3093 & 3095 & & 3140 & 141 & 291 & 3292 \\
\hline 3.800 & 1160 & 1178 & 1372 & 1372 & 1408 & 1413 & 1420 & 1432 & 3011 & 3014 & 3093 & 3095 & 31 & 3140 & 3140 & 3294 & 3294 \\
\hline 3.900 & 1159 & 1177 & 1371 & 1371 & 1407 & 1413 & 1420 & 1432 & 3011 & 3013 & 3092 & 3094 & 3105 & 3139 & 3140 & 3295 & 3296 \\
\hline & 1157 & 1176 & 1371 & 1371 & 1407 & 1413 & 1420 & 1432 & 3010 & 3013 & 3092 & 3094 & & 3139 & 3139 & 3296 & 3297 \\
\hline
\end{tabular}




\section{Moments of Inertia}

The following table lists all the (reduced) moments of inertia for the transitional modes and the methyl rotations used for the calculation of the free rotor partition functions $q_{f r}$ (Equation 5). The Moments $I$ are given in SI-units! The last six columns refer to the calculation of the moments of inertia for the Me-GaMe system (see part 5 of the supplementary material).

\begin{tabular}{|c|c|c|c|c|c|c|c|c|c|c|c|c|c|}
\hline RC & $\mathbf{Z}$ & X1 & Y1 & $\mathbf{X} 2$ & Y2 & Ms & Ma & $\mathbf{M}$ & C-Ga-C & $M / / z$ & $M / I 2$ & $\mathbf{A}$ & B \\
\hline 1.900 & $5.047 \mathrm{E}-47$ & $3.573 \mathrm{E}-46$ & $8.077 \mathrm{E}-46$ & $3.222 \mathrm{E}-47$ & $3.309 \mathrm{E}-47$ & $5.009 \mathrm{E}-47$ & $4.841 \mathrm{E}-47$ & $5.078 \mathrm{E}-47$ & 118.1 & 0.03 & & 0.99 & 0.95 \\
\hline 1.995 & $5.093 \mathrm{E}-47$ & 3.484E-46 & $8.415 \mathrm{E}-46$ & $3.209 \mathrm{E}-47$ & $3.282 \mathrm{E}-47$ & $5.012 \mathrm{E}-47$ & $4.866 \mathrm{E}-47$ & $5.089 \mathrm{E}-47$ & 120.4 & 0.03 & 0.03 & 0.98 & 0.96 \\
\hline 2.000 & $5.095 \mathrm{E}-47$ & $3.483 \mathrm{E}-46$ & 8.433E-46 & $3.208 \mathrm{E}-47$ & $3.280 \mathrm{E}-47$ & $5.013 \mathrm{E}-47$ & 4.867E-47 & $5.090 \mathrm{E}-47$ & 120.4 & 0.03 & 0.03 & 0.98 & 0.96 \\
\hline 2.100 & $5.137 \mathrm{E}-47$ & $3.396 \mathrm{E}-46$ & $8.789 \mathrm{E}-46$ & 3.194E-47 & $3.254 \mathrm{E}-47$ & $5.016 \mathrm{E}-47$ & $4.894 \mathrm{E}-47$ & $5.102 \mathrm{E}-47$ & 122.5 & 0.03 & 0.03 & 0.98 & 0.96 \\
\hline 2.200 & $5.178 \mathrm{E}-47$ & 3.356E-46 & $9.147 \mathrm{E}-46$ & $3.179 \mathrm{E}-47$ & 3.228E-47 & $5.022 \mathrm{E}-47$ & $4.920 \mathrm{E}-47$ & $5.114 \mathrm{E}-47$ & 123.9 & 0.03 & 0.03 & 0.98 & 0.96 \\
\hline 2.250 & $5.198 \mathrm{E}-47$ & $3.343 \mathrm{E}-46$ & $9.325 \mathrm{E}-46$ & $3.171 \mathrm{E}-47$ & $3.216 \mathrm{E}-47$ & $5.026 \mathrm{E}-47$ & $4.933 \mathrm{E}-47$ & $5.120 \mathrm{E}-47$ & 124.4 & 0.03 & 0.03 & 0.98 & 0.96 \\
\hline 2.300 & $5.217 \mathrm{E}-47$ & 3.333E-46 & $9.502 \mathrm{E}-46$ & $3.164 \mathrm{E}-47$ & $3.204 \mathrm{E}-47$ & $5.030 \mathrm{E}-47$ & $4.944 \mathrm{E}-47$ & $5.125 \mathrm{E}-47$ & 124.8 & 0.03 & 0.03 & .98 & .96 \\
\hline 2.400 & $5.255 \mathrm{E}-47$ & $3.318 \mathrm{E}-46$ & $9.851 \mathrm{E}-46$ & $3.148 \mathrm{E}-47$ & $3.181 \mathrm{E}-47$ & $5.037 \mathrm{E}-47$ & $4.968 \mathrm{E}-47$ & $5.137 \mathrm{E}-47$ & 125.7 & 0.03 & 0.02 & .98 & 0.97 \\
\hline .500 & $292 \mathrm{E}-47$ & 3.307E-46 & $1.019 \mathrm{E}-45$ & $3.132 \mathrm{E}-47$ & $3.159 \mathrm{E}-47$ & E-47 & $4.989 \mathrm{E}-47$ & $5.148 \mathrm{E}-47$ & & 0. & & 98 & .97 \\
\hline 2.600 & $5.327 \mathrm{E}-47$ & $3.301 \mathrm{E}-46$ & $1.053 \mathrm{E}-45$ & 3.11 & & & & & & & & & .97 \\
\hline 2.700 & $5.361 \mathrm{E}-47$ & $3.300 \mathrm{E}-46$ & $1.085 \mathrm{E}-45$ & $3.100 \mathrm{E}-47$ & & & & & & & & & .97 \\
\hline 2.750 & $378 \mathrm{E}-47$ & $3.301 \mathrm{E}-46$ & $1.101 \mathrm{E}-45$ & $3.092 \mathrm{E}-47$ & & & & & & & & 98 & 0.97 \\
\hline 2.800 & $5.394 \mathrm{E}-47$ & 3.30 & & & & & & & & & & & .97 \\
\hline 2.900 & $5.425 \mathrm{E}-47$ & 3.309 & $1.148 \mathrm{E}-45$ & 3.06 & 3.08 & & & 5.1 & & & & & .98 \\
\hline 3.000 & $5.454 \mathrm{E}-47$ & $3.320 \mathrm{E}-46$ & $1.177 \mathrm{E}-45$ & & & & & & & & & .98 & 0.98 \\
\hline 3.050 & $5.468 \mathrm{E}-47$ & 3.32 & 1.1 & & & & & & & & & & .98 \\
\hline 3.100 & $5.481 \mathrm{E}-47$ & 3.334E-46 & $1.206 \mathrm{E}-45$ & $3.042 \mathrm{E}-47$ & E-47 & $E-47$ & & 5.2 & 128.3 & 0.03 & & & .98 \\
\hline 3.150 & $5.494 \mathrm{E}-47$ & 3.342 & 1.22 & & & & & & & & & 0.98 & 0.98 \\
\hline 3.200 & $507 \mathrm{E}-47$ & 3.35 & 1.23 & & & & & & & & & 0.98 & .98 \\
\hline 3.250 & $532 \mathrm{E}-47$ & 3.43 & & & & & & & & & & & 0.98 \\
\hline 3.300 & $554 \mathrm{E}-47$ & 3.51 & 1.2 & & & & & & 126 & $0 .($ & & 0.98 & 0.98 \\
\hline 3.400 & $591 \mathrm{E}-47$ & 3.96 & 1.29 & & & & & & & & 2 & 0.98 & 0.98 \\
\hline 3.500 & EE-47 & 4.07 & & & & & & & & & & & 0.98 \\
\hline 3.600 & 5.642 & 4.16 & & & & & & & & & 1 & .98 & .98 \\
\hline 3.700 & $659 \mathrm{E}-47$ & 4.24 & & & & & & & & & 0.0 & 0.99 & 0.98 \\
\hline 3.750 & $5.666 \mathrm{E}-47$ & $4.27 \varepsilon$ & & & & & & & & & & & 0.98 \\
\hline 3.800 & $5.672 \mathrm{E}-47$ & 4.310 & & & & & & & & & & 0.9 & 0.98 \\
\hline 3.850 & $677 \mathrm{E}-47$ & $4.340 \mathrm{E}-46$ & 1.39 & 2.95 & 2.9 & 5.1 & & & & 0. & 0. & 0.99 & 0.98 \\
\hline 3.900 & & 4.367E-46 & & & & & & & & & & & 0.98 \\
\hline 3.950 & $5.685 \mathrm{E}-47$ & 4.393 & & & & & & & & & & & 0.98 \\
\hline 4.000 & $5.688 \mathrm{E}-47$ & 4.417E-46 & $1.431 \mathrm{E}-45$ & $2.949 \mathrm{E}-47$ & $2.950 \mathrm{E}-47$ & $6 \mathrm{E}-47$ & & $5.239 \mathrm{E}$ & & 0. & 0. & 0.99 & 0.98 \\
\hline 4.100 & $5.692 \mathrm{E}-47$ & $4.461 \mathrm{E}-46$ & & & & & & & & & & & 0.98 \\
\hline 4.300 & $5.696 \mathrm{E}-47$ & 4.534 & & & & $E-47$ & & & & & & 0.99 & 0.98 \\
\hline 4.500 & $5.698 \mathrm{E}-47$ & $4.596 \mathrm{E}-46$ & $1.522 \mathrm{E}-45$ & $2.946 \mathrm{E}-47$ & $2.948 \mathrm{E}-47$ & $5.180 \mathrm{E}-47$ & $5.173 \mathrm{E}-47$ & $5.251 \mathrm{E}-47$ & & 0. & 0.0 & 0.99 & 0.99 \\
\hline 4.750 & $5.706 \mathrm{E}-47$ & $4.673 \mathrm{E}-46$ & $1.559 \mathrm{E}-45$ & $2.947 \mathrm{E}-47$ & $2.948 \mathrm{E}-47$ & $5.186 \mathrm{E}-47$ & & & & & 0.01 & 0.99 & 0.99 \\
\hline 5.000 & $6.016 \mathrm{E}-47$ & $5.496 \mathrm{E}-46$ & $1.493 \mathrm{E}-45$ & $2.950 \mathrm{E}-47$ & $2.989 \mathrm{E}-47$ & $5.195 \mathrm{E}-47$ & $5.195 \mathrm{E}-47$ & $5.262 \mathrm{E}-47$ & 117.7 & 0.03 & 0.01 & 0.99 & 0.99 \\
\hline
\end{tabular}
sigma: 3

\begin{tabular}{|c|c|c|c|}
\hline rc & Mz & M & \\
\hline 900 & $1.66 \mathrm{E}-045$ & $1.69 \mathrm{E}-045$ & $3.19 \mathrm{E}-045$ \\
\hline & $1.71 \mathrm{E}-045$ & $1.73 \mathrm{E}-045$ & \\
\hline o & $1.72 \mathrm{E}-045$ & $1.73 \mathrm{E}-045$ & \\
\hline 100 & 1.76 & -045 & 3.3 \\
\hline 200 & 1.7 & -045 & \\
\hline 250 & 1.78 & -045 & 3. \\
\hline & 1.7 & 045 & \\
\hline 400 & 1.8 & 045 & \\
\hline 500 & 1.8 & 2.1 & \\
\hline .600 & $1.82 \mathrm{E}-045$ & $2.27 \mathrm{E}-045$ & 3E-045 \\
\hline 2.700 & $1.82 \mathrm{E}-045$ & $2.39 \mathrm{E}-045$ & $4.06 \mathrm{E}-045$ \\
\hline 2.750 & 1.83 & $5 E-045$ & $\mathrm{~F}-045$ \\
\hline 2.800 & 1.83 & $2.51 \mathrm{E}-045$ & $4.18 \mathrm{E}-045$ \\
\hline .900 & $1.84 \mathrm{E}-0$ & $2.64 \mathrm{E}-045$ & $4.32 \mathrm{E}-045$ \\
\hline .000 & $1.84 \mathrm{E}-045$ & $2.77 \mathrm{E}-045$ & $4.45 \mathrm{E}-045$ \\
\hline & $1.84 \mathrm{E}-04$ & $2.84 \mathrm{E}-045$ & \\
\hline & 1.84 & $2.91 \mathrm{E}-045$ & 4.59 \\
\hline & $1.84 \mathrm{E}-045$ & $2.98 \mathrm{E}-045$ & $4.67 \mathrm{E}-045$ \\
\hline
\end{tabular}$$
1
$$$$
3
$$

Table 3.2 Global rotations moments of inertia (in SI-units).

$\begin{array}{rc}\text { rc } & \text { Mz } \\ \mathbf{3 . 2 0 0} & 1.85 \mathrm{E}-045 \\ \mathbf{3 . 2 5 0} & 1.84 \mathrm{E}-045 \\ \mathbf{3 . 3 0 0} & 1.83 \mathrm{E}-045 \\ \mathbf{3 . 4 0 0} & 1.77 \mathrm{E}-045 \\ \mathbf{3 . 5 0 0} & 1.76 \mathrm{E}-045 \\ \mathbf{3 . 6 0 0} & 1.76 \mathrm{E}-045 \\ \mathbf{3 . 7 0 0} & 1.75 \mathrm{E}-045 \\ \mathbf{3 . 7 5 0} & 1.75 \mathrm{E}-045 \\ \mathbf{3 . 8 0 0} & 1.75 \mathrm{E}-045 \\ \mathbf{3 . 8 5 0} & 1.75 \mathrm{E}-045 \\ \mathbf{3 . 9 0 0} & 1.74 \mathrm{E}-045 \\ \mathbf{3 . 9 5 0} & 1.74 \mathrm{E}-045 \\ \mathbf{4 . 0 0 0} & 1.74 \mathrm{E}-045 \\ \mathbf{4 . 1 0 0} & 1.74 \mathrm{E}-045 \\ \mathbf{4 . 3 0 0} & 1.74 \mathrm{E}-045 \\ \mathbf{4 . 5 0 0} & 1.73 \mathrm{E}-045 \\ \mathbf{4 . 7 5 0} & 1.73 \mathrm{E}-045 \\ \mathbf{5 . 0 0 0} & 1.79 \mathrm{E}-045 \\ \mathbf{1 0 . 0 0 0} & 1.97 \mathrm{E}-045\end{array}$

Mx 3.05E-045 4.74E-045 3.14E-045 4.82E-045 3.23E-045 4.90E-045 3.47E-045 5.08E-045 3.64E-045 5.24E-045 $3.82 \mathrm{E}-045 \quad 5.41 \mathrm{E}-045$ $3.99 \mathrm{E}-045 \quad 5.58 \mathrm{E}-045$ 4.08E-045 5.67E-045 $4.17 \mathrm{E}-045 \quad 5.75 \mathrm{E}-045$ 4.26E-045 5.84E-045 4.35E-045 5.93E-045 $4.44 \mathrm{E}-045 \quad 6.02 \mathrm{E}-045$ $4.54 \mathrm{E}-045 \quad 6.11 \mathrm{E}-045$ 4.73E-045 6.30E-045 5.12E-045 6.69E-045 $5.52 \mathrm{E}-045 \quad 7.09 \mathrm{E}-045$ $6.05 \mathrm{E}-045 \quad 7.62 \mathrm{E}-045$ $6.55 \mathrm{E}-045 \quad 8.05 \mathrm{E}-045$ $2.28 \mathrm{E}-044 \quad 2.41 \mathrm{E}-044$ 


\section{Free Energies $G(r c)$}

The following table lists the free energies (3N-7 degrees of freedom) along the reaction coordinate relative to the initial minimum ( $\mathrm{rc}=1.995 ; 3 \mathrm{~N}-6$ degrees of freedom).

The column titles refer to the temperature in Kelvin. The first column (' 0 ”) thus gives $\mathrm{E}_{0 \mathrm{~K}}$ (corrected $\mathrm{E}_{\mathrm{DFT}}$ including ZPE).

\begin{tabular}{|c|c|c|c|c|c|c|c|c|c|c|c|c|c|c|c|}
\hline Rc & 0 & 400 & 450 & 500 & 600 & 700 & 800 & 900 & 1000 & 1100 & 1200 & 1300 & 1400 & 1500 & 160 \\
\hline 1.900 & 7.5 & 3.4 & 3.5 & 3.7 & 4.1 & 4.7 & 5.4 & 6.1 & 7.0 & 7.9 & 8.9 & 10.0 & 11.1 & 12.3 & \\
\hline 1.995 & 0.0 & -2.8 & -2.6 & -2.4 & -1.8 & -1.1 & -0.3 & 0.7 & 1.7 & 2.7 & 3.9 & 5.1 & 6.4 & 7.7 & \\
\hline 00 & 0.0 & -2 & -2 . & -2 & -1.7 & -1.0 & -0.2 & 0.7 & 1.7 & 2.8 & & .2 & 6.5 & 7.8 & \\
\hline 2.100 & 5.9 & 4.0 & 4.2 & 4.5 & 5.1 & 5.9 & 6.8 & 7.8 & 8.8 & 10.0 & 11.2 & 12.5 & & 15.2 & \\
\hline 2.200 & 20.5 & 18.9 & 19.1 & 19.4 & 20.0 & 20.7 & 21.5 & 22.5 & 23.5 & 24.6 & & & & & \\
\hline 2.250 & 30.1 & 28.6 & 28.8 & 29.0 & 29.6 & 30.2 & 31.0 & 31.9 & 32.9 & 33.9 & & 6.2 & & 38.7 & \\
\hline 2.300 & 40.6 & 39.2 & 39.4 & 39.6 & 40.1 & 40.8 & 41.5 & 42.3 & 43.2 & 44.2 & & 46.4 & 47.6 & 48.8 & \\
\hline 2.400 & 63.8 & 62.6 & 62.7 & 62.9 & 63.3 & 63.8 & 64.4 & 65.1 & 65.9 & 66.7 & 67.7 & 68.7 & 69.7 & 70.8 & \\
\hline 2.500 & 88.4 & 87.4 & 87.5 & 87.6 & 87.9 & 88.2 & 88.7 & 89.3 & 89.9 & 90.6 & 91 & 92.2 & 93.1 & 94.1 & \\
\hline 2.600 & 113.8 & 112.6 & 112.6 & 112.6 & 112.8 & 113.0 & 113.3 & 113.7 & & 114.7 & & 116.0 & & 17.5 & \\
\hline 2.700 & 39.3 & & 137.4 & & 137.3 & & & & & 8.3 & & .3 & & & \\
\hline 2.750 & 151.8 & & & & & & & & & & & & & & \\
\hline 2.800 & 164. & & & & & & & & & & & & & & \\
\hline 2.90 & 187.6 & & & & & & & & & & & & & & \\
\hline 3.000 & 210.0 & & & & & & & & & & & & & & \\
\hline 3.050 & 220.6 & 6.7 & 216.4 & & 15.3 & 14.7 & & & & 2.9 & & & & & \\
\hline 3.100 & 231.0 & 26.8 & 226.4 & 6.0 & 225.2 & 24.5 & 3.8 & 23.2 & 222.7 & 222.3 & 221.9 & 221.6 & 1.4 & 221.2 & \\
\hline 3.150 & 241.0 & 6.6 & 6.1 & 5.6 & 34.7 & 33.9 & 3.2 & 32.5 & 31.9 & 31.3 & 30.8 & 0.4 & & 9.7 & \\
\hline 3.200 & 250.7 & & 5.4 & & & & & & & & & & & & \\
\hline 3.25 & 259.6 & & & & & & & & & & & & & & \\
\hline & & & & & & & & & & & & & & & \\
\hline & & & & & & & & & & & & & & & \\
\hline & & & & & & & & & & & & & & & \\
\hline & 293.9 & & & & & & & & & & & & & & \\
\hline 3.700 & 299.4 & & & & & 274.4 & & & & & & & & & \\
\hline 3.750 & 301.7 & 37.9 & 5.8 & 3.6 & 79.3 & 274.9 & 0.5 & & & 57.7 & & 9.5 & 5.5 & 241.5 & \\
\hline 3.800 & 303.7 & 39.1 & 6.8 & 4.5 & 79.8 & 75.1 & 0.4 & & & & & & & 9.3 & \\
\hline 3.850 & 305.5 & 90.2 & 287.7 & & 80.3 & 275.3 & 0.3 & 5.4 & & 55.8 & & & & 237.4 & \\
\hline 3.900 & 307.0 & 291.1 & 288.5 & 85.9 & 280.7 & 275.4 & 270.2 & 5.1 & & 55.0 & & & & & \\
\hline 3.950 & 308.4 & 91.9 & 289.2 & & 281.1 & 275.7 & & & & & & & & & \\
\hline 4.000 & 309.7 & 292.6 & & & & & & & & & & & & & \\
\hline & & & & & & & & & & 252.7 & & & & 230.6 & 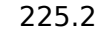 \\
\hline & 315.1 & 294.4 & & & 281.3 & 274.7 & & & & & & & & & \\
\hline & 317.2 & & 290.2 & & 279.1 & 271.8 & & & & & & & & 5.8 & \\
\hline & 318.9 & 291.9 & & & & & & & & & & & & & \\
\hline & & & & & & & & & & & & & & & \\
\hline & 320.8 & 278.6 & 273.0 & 267.4 & & 245.6 & 235.0 & 224.6 & 214.5 & 204.6 & 194.8 & 185.3 & 175.9 & 166.7 & \\
\hline
\end{tabular}

These values have been calculated by an automatic procedure, using a spreadsheet, that recalculates all the relevant partition functions from the input data according to a specified temperature value. This spreadsheet (OpenOffice 1.1) can be received from the authors upon request. 


\section{Calculation of the moments of inertia for the spectator methyl groups in fragment 1}

In Table 3.1 the moment of inertia for one of the two (symmetrically equivalent) methyl rotors in the Me-Ga-Me fragment 1 are given in the column "M". The corresponding moments for the symmetric ("Ms") and antisymmetric ("Ma") motion have been calculated following David Smith ("Tunneling frequencies of solids with two equivalent internal $\mathrm{C}_{3 v}$ rotors", J. Chem. Phys. 1980, 73, 3947.) for two $\mathrm{C}_{3 \mathrm{~V}}$ tops in a $\mathrm{C}_{\mathrm{S}}$ frame.

The following formulas have been used:

$$
\begin{aligned}
& \mathrm{Ms}=\mathrm{M} * \mathrm{~A} \\
& \mathrm{Ma}=\mathrm{M} * \mathrm{~B}
\end{aligned}
$$

with:

$$
\begin{aligned}
& \mathrm{A}=1-2 \cos (\alpha / 2)^{2} \mathrm{M} / \mathrm{Mz} \\
& \mathrm{B}=1-2 \sin (\alpha / 2)^{2} \mathrm{M} / \mathrm{Mx}
\end{aligned}
$$

where $\alpha$ is the $\mathrm{C}-\mathrm{Ga}-\mathrm{C}$ angle.

Both A, B and are also given in Table 3.1. Note that Mx and Mz refer to the moments of inertia for the external rotations around the principal axes given in Table 3.2.

\section{Calculation of the lambda parameter describing the mixing between ma and $x 1$}

Due to a mixing of the motions ma and $\mathbf{x} 1$ around $\mathrm{rc}=3.85 \AA$ we had to introduce an interpolation scheme for the calculation of the corresponding free rotor partition functions (see Equation 6). For this a parameter $\lambda$ is necessary, which goes from zero to one transforming normal mode 1 from $\mathbf{x} \mathbf{1}$ to ma and mode 2 from ma to $\mathbf{x} 1$.

This parameter has been determined as follows:

With the so called "potential energy distribution" (Y. Morino, K. Kuchitsu "Note on the classification of normal vibrations of molecule", J. Chem. Phys. 1952, 20, 1809.) the relative percentage of individual internal coordinates (representing $\mathbf{x} 1$ and $\mathbf{m a}$ ) to the two respective normal modes have been analyzed in the range from $\mathrm{rc}=3.6$ up to $4.1 \AA$, which gave two different lambda values (see Table 6.1). We used a fortran program originally written by V. A. Sipachev (V. A. Sipachev, J. Mol. Struct. 1985, 121, 143.), which was extended to directly read Gaussian98 output by M. Tafipolsky (M. Tafipolsky, private communication). The entry in "lambda 1" refers to the relative contribution of $\mathbf{m s}$ to mode 1 and "1 ambda 2 " to the relative contribution of $\mathbf{x} \mathbf{1}$ to mode 2 .

Table 6.1

$\begin{array}{crrrrrrrrr}\text { rc } & \mathbf{3 . 6} & \mathbf{3 . 7} & \mathbf{3 . 7 5} & \mathbf{3 . 8} & \mathbf{3 . 8 5} & \mathbf{3 . 9} & \mathbf{3 . 9 5} & \mathbf{4} & \mathbf{4 . 1} \\ \text { lambda 1 } & 0.17 & 0.29 & 0.36 & 0.47 & 0.57 & 0.66 & 0.73 & 0.79 & 0.88 \\ \text { lambda 2 } & 0.18 & 0.26 & 0.31 & 0.37 & 0.47 & 0.52 & 0.59 & 0.65 & 0.76 \\ \text { lambda(aver.) } & 0.17 & 0.27 & 0.33 & 0.42 & 0.52 & 0.59 & 0.66 & 0.72 & 0.82\end{array}$


These values were averaged (last row of Table 6.1) and fitted by an analytic function

$$
\lambda(\mathrm{rc})=0.5+0.5 * \operatorname{erf}(\mathrm{a} 1 *(\mathrm{rc}-\mathrm{a} 0))
$$

The values for the two fitting parameters a 0 and a1 are shown in Figure 6.1 together with the resulting smooth curve for the lambda parameter, which has been used for the mixing of the free rotor partition functions according to Equation 6 in the paper.

It should be noted (as stated also in Section 3.3) this procedure is not really physically grounded. It just avoids an abrupt change in the partition function leading to a kink of about $2 \mathrm{~kJ} / \mathrm{mol}$ size in the $\mathrm{G}$ curves. Thus it basically smoothes out this unwanted artifact. The values of $\mathrm{G}$ are changed by less then $1 \mathrm{~kJ} / \mathrm{mol}$ and the quality of this fitting procedure or the assignment of the lambda values do not affect the final results of the overall calculation.

Figure 6.1. Calculated averaged lambda values and fitted function.

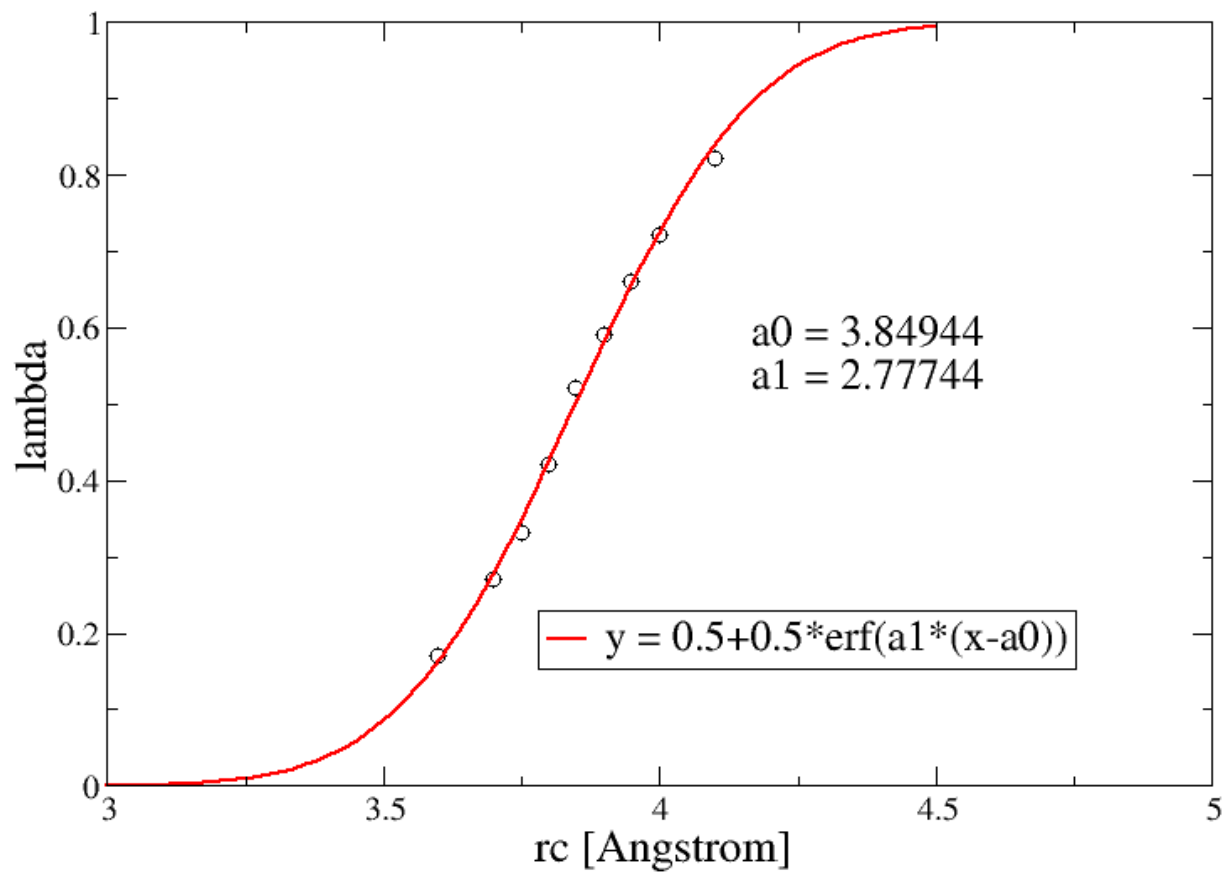

